\title{
Tranexamic acid decreases the risk of revision for acute and delayed periprosthetic joint infection after total knee replacement
}

\author{
Marek Lacko, MD, $\mathrm{PhD}^{1}$ (D), Pavol Jarčuška, MD, $\mathrm{PhD}^{2}$ (D), Daniela Schreierova, $\mathrm{MD}^{1}$ (D), \\ Antónia Lacková, $\mathrm{MD}^{3} \odot$, Ahmad Gharaibeh, MD, PhD, MPH' \\ 1'Department of Orthopedics and Traumatology of Locomotors Apparatus, Medical Faculty of P. J. Safarik University and University Hospital of \\ L. Pasteur, Kosice, Slovakia \\ ${ }^{2}$ Department of Infectology and Travel Medicine, Medical Faculty of P. J. Safarik University and University Hospital of L. Pasteur, Kosice, Slovakia \\ ${ }^{3}$ Department of Neurology, University Hospital of L. Pasteur, Kosice, Slovakia
}

Total knee replacement (TKR) is an effective treatment option for advanced osteoarthritis (OA) of the knee, which decreases pain and improves function. ${ }^{[1]}$ Nevertheless, some patients achieve poor results after surgery or the implant fails and a revision surgery is required.

The main cause of TKR revisions in the first three years after primary surgery is periprosthetic joint infection (PJI). ${ }^{[2,3]}$ Periprosthetic joint infection is classified according to when symptoms start after TKR as early, delayed and late. ${ }^{[4]}$ Early infections occur within three months of TKR, delayed between 3 and 24 months and late from 24 months after TKR. Because of the severe morbidity and costs associated with TKR revisions for PJI, we need to focus on preventing PJIs.

Tranexamic acid (TA) is a synthetic antifibrinolytic substance that inhibits proteolytic degradation of fibrin by blocking the lysine binding sites of

Received: November 06, 2019

Accepted: January 01, 2020

Published online: March 02, 2020

Correspondence: Marek Lacko, MD. Department of Orthopedics and Traumatology of Locomotors Apparatus, Medical Faculty of P. J. Safarik University and University Hospital of L. Pasteur, Trieda SNP 1, 04001 Kosice, Slovakia.

E-mail: marek.lacko@upjs.sk

Doi: $10.5606 /$ ehc. 2020.72061

Citation: Lacko $M$, Jarčuška $P$, Schreierova $D$, Lacková $A$, Gharaibeh A. Tranexamic acid decreases the risk of revision for acute and delayed periprosthetic joint infection after total knee replacement. Jt Dis Relat Surg 2020;31(1):8-13

\section{ABSTRACT}

Objectives: This study aims to analyze the effect of intravenous administration of tranexamic acid (TA) on reducing the risk of revision for acute and delayed periprosthetic joint infection (PJI) after primary total knee replacement (TKR).

Patients and methods: This prospective observational cohort study included 1,529 TKRs (396 males, 1,133 females; mean age 67.8 years; range, 44 to 85.1 years) performed between January 2003 and October 2017. We analyzed the revision rate for acute and delayed PJI in a group of 787 TKRs with preoperatively intravenously administered TA (TA group) in comparison with a group of 742 TKRs without administration of TA (non-TA group). Multiple logistic regression analysis was used to evaluate significant predictors of TKR revision for acute and delayed PJI.

Results: Revision surgery due to PJI was recorded in one patient in the TA group and eight patients in the non-TA group. Cumulative revision rate of TKR was significantly lower in the TA group $(0.13 \%$ vs. $1.08 \%$, hazard ratio $0.113 ; 95 \%$ confidence interval [CI] 0.0147-0.937; $\mathrm{p}=0.043$ ). Multivariate logistic regression analysis confirmed two predictors of revision: being aged over 75 years at the time of primary surgery (odds ratio [OR] $8.464 ; 95 \%$ CI: 2.016-35.54; $\mathrm{p}=0.004)$ and male gender (OR: 7.9; 95\% CI: $1.879-33.26 ; \mathrm{p}=0.005)$. The use of TA was shown as the significant protective factor (OR: 0.109; 95\% CI: 0.0128-0.929; $\mathrm{p}=0.043$ ).

Conclusion: We have found a lower cumulative revision rate of TKR for acute and delayed PJI when TA was used. We think that the preoperative intravenous use of TA may be an effective, safe and inexpensive method for the prevention of PJI.

Keywords: Knee replacement, periprosthetic joint infection, revision, tranexamic acid.

plasminogen, thereby preventing plasminogen activation and binding of plasmin to fibrin. Intravenous or topical administration of TA seems to be an effective method in the reduction of blood loss and blood transfusions following TKR. ${ }^{[5]}$ The 
allogenic blood transfusion causes alteration in immune system which can result in higher risk of surgical site infection. ${ }^{[6-8]}$ Thus TA may reduce the rate of early and delayed PJI. In this study, we aimed to analyze the effect of intravenous administration of TA on reducing the risk of revision for acute and delayed PJI after primary TKR.

\section{PATIENTS AND METHODS}

This prospective observational cohort single-center study identified 1,571 cemented cruciate retaining TKRs without patellar resurfacing performed at Department of Orthopedics and Traumatology of Locomotors Apparatus, Medical Faculty of P. J. Safarik University and University Hospital of L. Pasteur, Kosice, Slovakia, between January 2003 and October 2017. We included in the analysis 1,529 primary TKRs (396 males, 1,133 females; mean age 67.8 years; range, 44 to 85.1 years) with a minimal follow-up duration of 24 months. Due to insufficient follow-up clinical data, we excluded 42 patients. Subsequent revision surgery for acute or delayed PJI was recorded in nine patients. The study protocol was approved by the University Hospital of L. Pasteur in Kosice Ethics Committee (EK22022018). A written informed consent was obtained from each patient. The study was conducted in accordance with the principles of the Declaration of Helsinki.

The selected set of primary TKRs was divided into analysis groups as the TA group and non-TA group according to whether TA was administered during implantation surgery. Patients of TA-group received one dose of $10 \mathrm{mg} / \mathrm{kg}$ TA 20 minutes prior to skin incision.

We have been using TA in TKR since 2012. The rationale for using TA was based on a published result of a meta-analysis which confirmed the efficacy and safety of TA in reducing the blood loss involved in TKR..$^{[9]}$ We indicated usage of TA regardless of the age and concomitant diseases of patients.

We summarized and analyzed all revisions of TKRs due to PJI in a period of 24 months after primary surgery. The revision of TKR was defined as the reoperation of knee arthroplasty with the replacement, removal or addition of one or more components or any reoperation. Periprosthetic joint infection was defined by the Musculoskeletal Infection Society criteria. ${ }^{[10]}$ Acute PJI was defined if infection was diagnosed within three months, while delayed PJI was defined if symptoms of infection occurred between 3 and 24 months after primary replacement.
The age, gender and body mass index (BMI) of patients, the etiology of $\mathrm{OA}$ of the operated knee (primary OA, posttraumatic OA, rheumatoid arthritis [RA]), the presence of diabetes mellitus (DM), the number of blood transfusion units used during primary TKR and the time to revision surgery were recorded.

The cumulative percent revision for acute or delayed PJI was compared between the groups over the same time period. Multiple logistic regression analysis was used to evaluate significant predictors of TKR revision for acute and delayed PJI. The effect of individual independent variables (use of TA; being aged lower than 55 years at the time of primary surgery; being aged higher than 75 years at the time of primary surgery; male gender; BMI higher than $40 \mathrm{~kg} / \mathrm{m}^{2}$; post-traumatic OA; RA; DM) on the observed event, i.e. dependent variable-revision surgery of TKR was analyzed.

The data for analysis were collected from the individual search of patients' medical documentation and verified with reports from national arthroplasty register, which additionally identified one revision performed at an institution outside the study hospital.

All operations were performed using the same surgical technique (midline skin incision, medial parapatellar capsulotomy, intramedullary aiming for femoral resection and extramedullary aiming for tibial resection). The cemented posterior cruciate ligament retaining knee replacement was used in all patients. Evacuation drainage was left on for 48 hours. Antibiotic prevention (second-generation cephalosporin, or clindamycin if the patient had an allergy to beta-lactamases) and prevention of thromboembolism (low-molecular-weight heparin) were the same in all patients.

\section{Statistical analysis}

Student's t-test was used for statistical analysis of continuous variables. If the files had an abnormal distribution, the Mann-Whitney $U$ test was used for the analysis. Frequency of data was judged using the chi-squared test.

The unadjusted cumulative percent revision at the end of the first two years after the primary arthroplasty, with an accompanying $95 \%$ confidence interval (CI), was calculated with use of unadjusted pointwise Greenwood estimates. The hazard ratio (HR) was calculated with use of Cox proportional hazards model and used to make statistical comparisons of the revision rates between the groups. The results of multivariate logistic regression analysis were reported 


\begin{tabular}{|c|c|c|c|c|c|c|c|c|c|}
\hline \multicolumn{10}{|c|}{$\begin{array}{c}\text { TABLE I } \\
\text { Univariate analysis }\end{array}$} \\
\hline & \multicolumn{4}{|c|}{ TA group $(n=787)$} & \multicolumn{4}{|c|}{ Non-TA group $(\mathrm{n}=742)$} & \multirow[b]{2}{*}{$p^{*}$} \\
\hline & $\mathrm{n}$ & $\%$ & Mean \pm SD & Min-Max & $\mathrm{n}$ & $\%$ & Mean $\pm S D$ & Min-Max & \\
\hline Mean age (year) & & & $68.1 \pm 6.6$ & $44-84.6$ & & & $67.4 \pm 7.2$ & $44-85.1$ & 0.09 \\
\hline \multicolumn{10}{|l|}{ Age categories } \\
\hline$<55$ years & 39 & 5 & & & 44 & 6 & & & 0.39 \\
\hline $55-75$ years & 609 & 77 & & & 545 & 73 & & & 0.07 \\
\hline$>75$ years & 139 & 18 & & & 153 & 21 & & & 0.14 \\
\hline Body mass index $\left(\mathrm{kg} / \mathrm{m}^{2}\right)$ & 31.3 & 3.9 & $20.4-44$ & & 30.9 & 4.9 & & $21.4-43.5$ & 0.45 \\
\hline \multicolumn{10}{|l|}{ Gender } \\
\hline Female & 581 & 74 & & & 552 & 75 & & & 0.64 \\
\hline Male & 206 & 26 & & & 190 & 25 & & & 0.65 \\
\hline \multicolumn{10}{|l|}{ Osteoarthritis } \\
\hline Primary & 697 & 88 & & & 659 & 89 & & & 0.54 \\
\hline Post-traumatic & 38 & 5 & & & 47 & 6 & & & 0.39 \\
\hline Rheumatic & 52 & 7 & & & 36 & 5 & & & 0.1 \\
\hline Diabetes mellitus & 51 & 7 & & & 47 & 6 & & & 0.43 \\
\hline Blood transfusion (units) & & & $0.52-0.65$ & $0-4$ & & & $1.41-1.03$ & $0-6$ & $<0.001$ \\
\hline
\end{tabular}

as odds ratio (OR) for revision, with a $95 \% \mathrm{CI}$ and a corresponding significance level (p). The level for statistical significance was set at $\mathrm{p}<0.05$ for all tests. SigmaPlot version 12.5 (Systat Software, Inc., San Jose, CA, USA) was used for statistical analyses.

\section{RESULTS}

The TA group consisted of 787 primary TKRs. A total of 742 TKRs were performed without administration of TA. No statistically significant differences were found between the analyzed groups with respect to the average age, gender and patients' BMI or the occurrence of post-traumatic and rheumatic OA and DM. Significantly higher amount of blood transfusion units was used in non-TA group (1.41 vs. 0.52 unit; $\mathrm{p}<0.001)$. Results of univariate analysis are shown in Table I.

In the TA group, we recorded one revision surgery due to delayed PJI. In the non-TA group, overall eight revisions were performed: one revision for acute PJI

\begin{tabular}{|c|c|c|c|c|c|c|c|c|c|}
\hline \multicolumn{10}{|c|}{ TABLE II } \\
\hline $\begin{array}{l}\text { Case } \\
\text { number }\end{array}$ & $\begin{array}{l}\text { Age } \\
\text { (year) }\end{array}$ & Gender & $\begin{array}{c}\text { BMI } \\
\left(\mathrm{kg} / \mathrm{m}^{2}\right)\end{array}$ & $\begin{array}{l}\text { Indication } \\
\text { diagnosis }\end{array}$ & $\begin{array}{l}\text { Diabetes } \\
\text { mellitus }\end{array}$ & $\begin{array}{c}\text { Use of } \\
\text { tranexamic } \\
\text { acid }\end{array}$ & $\begin{array}{l}\text { Blood transfusion } \\
\text { used (units) }\end{array}$ & $\begin{array}{l}\text { Type of } \\
\text { PJI }\end{array}$ & $\begin{array}{l}\text { Time to } \\
\text { revision } \\
\text { (month) }\end{array}$ \\
\hline 1 & 75.5 & Female & 33.7 & Primary $\mathrm{OA}$ & Yes & No & 2 & Delayed & 21.8 \\
\hline 2 & 79.4 & Male & 22.3 & Primary OA & No & No & 3 & Acute & 2.7 \\
\hline 3 & 74.8 & Female & 31.1 & Primary OA & No & No & 1 & Delayed & 20.6 \\
\hline 4 & 60.2 & Male & 28.1 & Primary $\mathrm{OA}$ & No & No & 0 & Delayed & 20.8 \\
\hline 5 & 81.4 & Male & 34.3 & Primary OA & No & No & 2 & Delayed & 18.2 \\
\hline 6 & 83.2 & Male & 30.1 & Primary OA & No & No & 1 & Delayed & 19.8 \\
\hline 7 & 69.2 & Male & 22.6 & Primary OA & No & Yes & 2 & Delayed & 10.3 \\
\hline 8 & 75.1 & Male & 33.2 & Primary OA & No & No & 2 & Delayed & 18.2 \\
\hline 9 & 67.2 & Female & 25.1 & $\begin{array}{l}\text { Rheumatoid } \\
\text { arthritis }\end{array}$ & No & No & 2 & Delayed & 11.9 \\
\hline
\end{tabular}




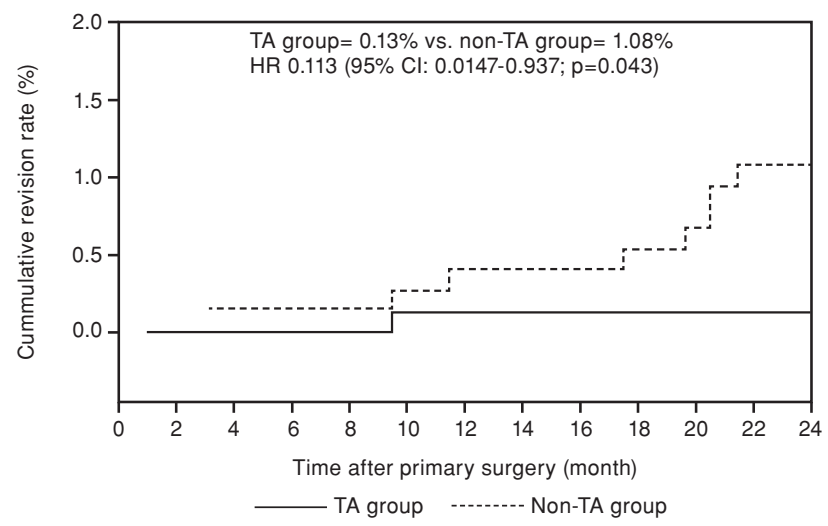

FIGURE 1. Cumulative percent revision for acute and delayed periprosthetic joint infection of primary total knee arthroplasty by tranexamic acid usage. There was a higher rate of revision when tranexamic acid was not used $(1.08 \%$ vs. $0.13 \% ; p<0.05)$.

TA: Tranexamic acid; HR: Hazard ratio; Cl: Confidence interval.

and seven revisions for delayed PJI (Table II). All cases of infected TKRs were treated with two-stage reimplantation surgery.

Cumulative revision rate of TKR was significantly lower in the TA group $(0.13 \%$ vs. $1.08 \%$; HR $0.113 ; 95 \%$ CI: 0.0147-0.937; $\mathrm{p}=0.043$; Figure 1).

Multivariate logistic regression analysis confirmed two negative predictors of revision: being aged over 75 years at the time of primary surgery (OR: 8.464; 95\% CI: 2.016-35.54; $\mathrm{p}=0.004$ ) and male gender (OR: 7.9; 95\% CI: 1.879-33.26; $\mathrm{p}=0.005)$. The use of TA was shown as the significant protective factor (OR: 0.109; 95\% CI: 0.0128-0.929; $\mathrm{p}=0.043$; Table 3).

\section{DISCUSSION}

During the entire observation period (2003-2017), we found a statistically significantly higher revision rate for acute and delayed PJI after TKR when TA was not administered. We confirmed in the multivariate analysis that use of TA was the protective factor for revision due to acute and delayed PJI.

Total knee replacement is an effective treatment option for advanced OA of the knee. Revision rates are generally low: the revision risk at 10 years as reported in the national arthroplasty registries is about 5\%. ${ }^{[11-13]}$ Half of these revisions occur within three years of the index primary TKR. The number of revisions is growing due to a growing number of overall TKRs, a shift towards younger patients and technical developments in revision implants.

The PJI is the main reason of failure and revision in TKR, with a PJI incidence of $1.0 \%$ at two years and $2.0 \%$ at 15 years after primary surgery. ${ }^{[2,3]}$ The PJI in the first two years after arthroplasty is usually related to surgical procedure; therefore, can be considered as a hospital-acquired infection.

Since PJI is the most challenging complication after TKR, orthopedic research should focus on prevention of infection. There is a large volume of research dedicated towards lowering the infection risk factors such as patient's optimization, efficient surgical technique, maintaining optimal intraoperative conditions and decreasing postoperative complications. ${ }^{[14]}$ Intravenous or topical administration of TA seems to be an effective and safe method for reduction of blood loss after TKR. ${ }^{[5]}$

\begin{tabular}{|c|c|c|c|c|}
\hline \multicolumn{5}{|c|}{$\begin{array}{l}\text { TABLE III } \\
\text { Multivariate logistic regression analysis }\end{array}$} \\
\hline Individual variable & $p$ & Odds ratio & $5 \%$ confidence lower & $95 \%$ confidence upper \\
\hline Constant & $<0.001$ & 0.00202 & 0.000389 & 0.0105 \\
\hline Age (<55 years) & 0.997 & 3.44E-07 & 0 & (+inf) \\
\hline Age (>75 years) & 0.004 & 8.464 & 2.016 & 35.54 \\
\hline \multicolumn{5}{|l|}{ Gender } \\
\hline Male & 0.005 & 7.906 & 1.879 & 33.26 \\
\hline Body mass index $\left(>40 \mathrm{~kg} / \mathrm{kg} / \mathrm{m}^{2}\right)$ & 0.998 & 2.07E-07 & 0 & (+inf) \\
\hline Posttraumatic osteoarthritis & 0.996 & $1.01 \mathrm{E}-07$ & 0 & (+inf) \\
\hline Rheumatoid arthritis & 0.243 & 3.72 & 0.41 & 33.724 \\
\hline Diabetes mellitus & 0.547 & 0.521 & 0.0623 & 4.357 \\
\hline Tranexamic acid & 0.043 & 0.109 & 0.0128 & 0.929 \\
\hline
\end{tabular}


In our study, we found that use of TA in primary TKR was associated with lower revision rate for acute and delayed PJI. To our knowledge, there is one other study that investigated the effect of TA on the incidence of PJI after TKR. In that retrospective study, Yazdi et al. ${ }^{[15]}$ concluded that TA could help to reduce the rate of PJI after primary TKR.

The advantage of TA administration on reducing the risk of revision for PJI is probably related to the reduction of blood loss. Decreasing perioperative blood loss can help to decrease postoperative hematoma formation and allogenic blood transfusion requirements. Several authors also confirmed the direct positive effect of TA on wound healing and skin regeneration. ${ }^{[16,17]}$ They showed that antifibrinolytic agents increase collagen synthesis and tensile strength within granulation tissue, presumably by preserving the fibrin matrix. Allogeneic blood transfusion is associated with an increased risk of surgical site infection and PJI. ${ }^{[6-8]}$ This has been hypothesized to be related to the immunomodulatory effects of transfusion. The results of our study confirm this hypothesis. ${ }^{[18]}$

On the other hand, our findings contradict with results of several studies describing the role of fibrinolysis in the biofilm formation. In an experimental animal study, Kwiecinski et al. ${ }^{[19]}$ found that precoating of the surface of indwelling medical devices with plasminogen activators resulted in less fibrin deposition on the implant surface which reduced the attachment of free-floating bacteria with subsequent biofilm formation. Similarly, Jørgensen et al. ${ }^{[20]}$ and Hogan et al. ${ }^{[21]}$ detected that biofilm dispersal fibrinolytic agents increase the penetration and effect of antibiotics in biofilm-related infections. Since TA has an anti-fibrinolytic effect, in the context of previously mentioned studies, TA administration should be associated with a higher risk of biofilm formation and subsequent acute and delayed PJI. This was not confirmed in our study. Biofilm formation is a complex process, so it is possible that there are other unknown interactions between TA and biofilm pathogenesis. Further research should be conducted on this subject to clarify the exact mechanism of the action of TA in relation to PJI.

There are other risk factors associated with PJI. The male gender, higher age, RA, post-traumatic OA and DM are patient-related risk factors of PJI. ${ }^{[22,23]}$ In the multivariate analysis, which included these factors and the use of TA as independent variables, we also identified the male gender and higher age as predictors of revision for acute and delayed PJI.
Analysis of other patient-related risk factors failed to reach the level of statistical significance.

This study has some limitations. Firstly, this study, which was based on the analysis of performed revisions of TKR, may not necessarily reflect the real number of infected joint replacements. Some cases of PJI may have been misdiagnosed as aseptic loosening of TKR or may never have surgical revision for various reasons. Moreover, there is no information on the completeness and correctness of our national arthroplasty register, thus some revised cases of PJI can be missed. Another limitation may be certain "inhomogeneity" of the evaluated group of TKRs. They included several models of prostheses that were implanted by several orthopedic surgeons with different levels of experience. However, cemented total knee prostheses were used in all cases preserving posterior cruciate ligament without primary patellar resurfacing divided into the evaluated groups with almost equal number of TKRs. All TKRs were performed by the similar surgical technique, with the same postoperative protocol.

In conclusion, PJI remains one of the major concerns in the field of knee arthroplasty. We have found a lower cumulative revision rate of TKR for acute and delayed PJI when TA was used. We think that the preoperative intravenous use of TA may be an effective, safe and in-expensive method for the prevention of PJI after TKR. Further randomized controlled studies should be set up to corroborate these findings.

\section{Declaration of conflicting interests}

The authors declared no conflicts of interest with respect to the authorship and/or publication of this article.

\section{Funding}

The work was supported by the Scientific Grant Agency of the Ministry of Education, Science, Research and Sports of the Slovak Republic - VEGA no.1/0598/20.

\section{REFERENCES}

1. Carr AJ, Robertsson O, Graves S, Price AJ, Arden NK, Judge A, et al. Knee replacement. Lancet 2012;379:1331-40.

2. Postler A, Lützner C, Beyer F, Tille E, Lützner J. Analysis of total knee arthroplasty revision causes. BMC Musculoskelet Disord 2018;19:55.

3. Lum ZC, Shieh AK, Dorr LD. Why total knees fail-A modern perspective review. World J Orthop 2018;9:60-4.

4. Zimmerli W, Trampuz A, Ochsner PE. Prosthetic-joint infections. N Engl J Med 2004;351:1645-54.

5. Fillingham YA, Ramkumar DB, Jevsevar DS, Yates AJ, Shores $\mathrm{P}, \mathrm{Mullen} \mathrm{K}$, et al. The efficacy of tranexamic acid in total knee arthroplasty: a network meta-analysis. J Arthroplasty 2018;33:3090-98.e1. 
6. Taneja A, El-Bakoury A, Khong H, Railton P, Sharma R, Johnston KD, et al. Association between allogeneic blood transfusion and wound infection after total hip or knee arthroplasty: A retrospective case-control study. J Bone Jt Infect 2019;4:99-105.

7. Youssef LA, Spitalnik SL. Transfusion-related immunomodulation: a reappraisal. Curr Opin Hematol 2017;24:551-7.

8. Viola J, Gomez MM, Restrepo C, Maltenfort MG, Parvizi J. Preoperative anemia increases postoperative complications and mortality following total joint arthroplasty. J Arthroplasty 2015;30:846-8.

9. Alshryda S, Sarda P, Sukeik M, Nargol A, Blenkinsopp J, Mason JM. Tranexamic acid in total knee replacement: a systematic review and meta-analysis. J Bone Joint Surg [Br] 2011;93:1577-85.

10. Parvizi J, Gehrke T. International consensus on periprosthetic joint infection: let cumulative wisdom be a guide. J Bone Joint Surg [Am] 2014;96:441.

11. Australian Orthopaedic Association National Joint Replacement Registry. Annual Report. Adelaide: AOA; 2018. https://aoanjrr.sahmri.com/annual-reports-2018

12. Registry NJ. National Joint Registry. $15^{\text {th }}$ Annual Report 2018 - National Joint Registry for England, Wales, Northern Ireland and the Isle of Man; 2018.

13. Registry SKA. The Swedish Knee Arthroplasty Register. Annual Report 2018. 2018.

14. Solarino G, Abate A, Vicenti G, Spinarelli A, Piazzolla A, Moretti B. Reducing periprosthetic joint infection: what really counts? Joints 2016;3:208-14.

15. Yazdi $H$, Klement MR, Hammad M, Inoue $D, X u C$, Goswami K, et al. Tranexamic acid is associated with reduced periprosthetic joint infection after primary total joint arthroplasty. J Arthroplasty 2019 Oct 22. pii: S08835403(19)31006-X.

16. Vinckier F, Vermylen J. Wound healing following dental extractions in rabbits: effects of tranexamic acid, warfarin anti-coagulation, and socket packing. J Dent Res 1984;63:646-9.

17. Björlin G, Nilsson IM. The effect of antifibrinolytic agents on wound healing. Int J Oral Maxillofac Surg 1988;17:275-6.

18. Atik OŞ. Is there something new and interesting in my article? Eklem Hastalik Cerrahisi 2019;30:69.

19. Kwiecinski J, Na M, Jarneborn A, Jacobsson G, Peetermans $\mathrm{M}$, Verhamme $\mathrm{P}$, et al. Tissue plasminogen activator coating on implant surfaces reduces Staphylococcus aureus Biofilm Formation. Appl Environ Microbiol 2015;82:394-401.

20. Jørgensen NP, Zobek N, Dreier C, Haaber J, Ingmer $H$, Larsen $\mathrm{OH}$, et al. Streptokinase treatment reverses biofilmassociated antibiotic resistance in Staphylococcus aureus. Microorganisms 2016;4. pii: E36.

21. Hogan S, O'Gara JP, O'Neill E. Novel treatment of Staphylococcus aureus device-related infections using fibrinolytic agents. Antimicrob Agents Chemother 2018;62. pii: e02008-17.

22. Kunutsor SK, Whitehouse MR, Blom AW, Beswick AD; INFORM Team. Patient-related risk factors for periprosthetic joint infection after total joint arthroplasty: A systematic review and meta-analysis. PLoS One. 2016 Mar 3;11:e0150866.

23. Kong L, Cao J, Zhang Y, Ding W, Shen Y. Risk factors for periprosthetic joint infection following primary total hip or knee arthroplasty: a meta-analysis. Int Wound J 2017;14:529-36. 